\title{
DYNAMICAL SYMMETRIES FOR FERMIONS
}

\author{
Mike GuidRY \\ Department of Physics, University of Tennessee, Knoxville, TN 37996-1200
}

\begin{abstract}
An introduction is given to the Fermion Dynamical Symmetry Model (FDSM). The analytical symmetry limits of the model are then applied to the calculation of physical quantities such as ground-state masses and $B(E 2)$ values in heavy nuclei. These comparisons with data provide strong support for a new principle of collective motion, the Dynamical Paxli Effect, and suggest that dynamical symmetries which properly account for the Pauli principle are much more persistent in nuclear structure than the corresponding boson symmetries. Finally, we present an assessment of criticisms which have been voiced concerning the FDSM, and a discussion of new phenomena and "exotic spectroscopy" which may be suggested by the model.
\end{abstract}

\section{INTRODUCTION}

I would like to introduce to you today a new approacls to nuclear structure physics. The method I wish to discuss

- Uses principles of dynamical symmetry, but has no bosons.

- Has no explicit deformations, or moments of inertia, or other macroscopic "spontaneous breakers of symmetry"; yet it reproduces the observable matrix elements of collective-model physics.

- Represents a solution to a problem generally thought intractable (the spherical shell model for heavy collective nuclei); yet in many cases the solutions are not only feasible, they are analytical.

- Is a model for which many physically interesting problems are so ridiculously simple that you can obtain approximate solutions with a hand calculator; nevertheless, in some cases these results are in better or comparable accord with data than largescale computer calculations with standard approaches.

- Represents a novel perspective; therefore, it suggests important new ways of understanding nuclear structure which may not easily be seen in standard models.

The model to which I refer is the Fermion Dynamical Symmetry Model (FDSM), of course. ${ }^{1,2}$ In the time that I have available, I would like to at least make plausible the above assertions, by providing a simple introduction to the basic features of the model, by applying the model to the calculation of physically interesting quantities in a variety of systems, by discussing (objectively, I hope) criticisms that have occasionally been leveled at the model, and finally - for the sake of our organizer - by addressing the question of "exotic spectroscopy".

Invited paper presented at Symposium on Exotic Spectroscopy; Miami, Florida, September, 1989. The research presented here has been done in collaboration with several people; I especially want to acknowledge the contribution of Cheng-Li Wu at the University of Tennessee and Drexel University, Xiao-Ling Han, and Zhen-Ping Li at the University of Tennessee, and D. H. Feng and J. Q. Chen at Drexel University. 


\section{A SUMMARY OF RECENT FDSM RESULTS}

Before introducing the FDSM, I want to motivate the discussion by providing a compressed summary of what I consider to be the most important accomplishments of the FDSM within the past four years. $\Lambda$ fter all, if you are going to be forced to learn something new, you have some right to demand that the new thing deliver some "bang for the buck", so to speak.

1. IBM is the large-degeneracy limit of FDSM - Phys. Rev. 34C, 2269 (1986) ; 37C, 2789 (1988).

2. Particle-Rotor Model is the large particle number limit of the FDSM - Phys. Lett. 193B, 163 (1987) ; 198B, 119 (1987).

3. HFB is coherent-state approximation to the FDSM in the truncated model space Phys. Rev. 37C, 1281 (1988)

4. The FDSM is identically the complete shell model if all heritages ("broken pairs") are included - Phys. Rev. 36C, 1157 (1987)

5. The Nilsson model is recovered for deformed nuclei - Phys. Rev. 37C, 1739 (1988)

6. The FDSM prescribes a connection, Valence Shell $\longrightarrow$ Dynamical Synmetry $\longrightarrow$ Dominant Collective Modes, which is supported by nuclear systematics - Phys. Lett. 192B, 253 (1987)

7. Three kinds of vibrations, differing in Pauli effects - Phys. Rev. 36C, 1157 (1987)

8. A new transitional symmetry, SO(7), predicted and observed - Phys. Rev. Lett. 56, 2578 (1986)

9. Two kinds of rotation:

- $S p(6) \supset S U(3) \quad$ axially symmetric

- $S O(8) \supset S O(6) \quad \gamma$-soft rotor

- Phys. Lett. 168B, 313 (1986)

10. $B(E 2)$ values in deformed nuclei comparable in accuracy with large-scale numerical calculations:

- Analytical $B\left(E 2 ; 2^{+} \rightarrow 0^{+}\right)$of all heavy deformed nuclei with 3 parameters - Phys. Lett. B205, 156 (1988)

- Analytical description of $E 2$ collectivity loss in heavy nuclei; algebraic description of band termination - Phys. Lett 187B, 210 (1987), and To be published

11. Analytical description of actinide masses comparable in accuracy with large-scale Strutinsky calculations - Phys. Lett. 194B, 447 (1987), and To be published

12. Shell-model derivation of the VMI effect: stretching and Coriolis antipairing - To be published

13. Shell-model description of basic high-spin phenomena: band crossings and backbending - Phys. Lett. 176B, 1 (1986)

14. Identification of a "new" principle in collective motion: The Dynamical Pauli Effect. Widespread evidence in spectra, transition matrix elements, and masses - Phys. Left. 205B, 156 (1988) 
15. Possible origin of Coriolis attenuation problem of Particle-Rotor Model in finite particle number effects - Phys. Rev. 37C, 1739 (1988)

16. Simple algebraic explanation for the dominance of prolate over oblate geometry in heavy deformed nuclei - Submitied to Phys. Lett. B

17. Relation of $\gamma$-instability in high-spin states to Pauli representation restrictions on the dynamical symmetry - Phys. Rev. 38C, 1475 (1988)

18. A preliminary FDSM effective interaction for actinide nuclei which gives a good description of a variety of observables:

- ground-state masses

- $2^{+}$energies

- odd-even mass differences ("pair gap")

- $\beta, \gamma$ hand-head energies

- energies of yrast states

- $B(E 2)$ values on yrast line

- To be published

19. Suggested connection between dynamical symmetry breaking and occurrence of quantum chaos in nuclear structure - Phys. Rev. Lett. 61, 2167 (1988)

20. A suggestion that ground-band fluctuations in $B(E 2)$ values by $5-10 \%$ from rotational are CAP effects - Phys. Lett. 176B, 1 (1986)

21. Prssible explanation of recently observed large suppression of 2-neutron transfer to $I \approx 5-10 \hbar$ states in heavy-ion collisions -To be published

22. Demonstration of equivalence of algebraic and geometrical descriptions of vibrational bands in deformed nuclei:

- FDSM $\underset{n \rightarrow \infty}{\longrightarrow}$ Alaga values for $B(E 2)$ ratios

- 1:1 correspondence in state labeling

- Submitted for Publication to Fizika

23. First stage of FDSM code (valid for no broken pairs) now available. Numerical calcuJation of observables [spectrum, $Q, B(E 2), B(M 1), \ldots$ ] away from symmetry limits. Stage 2 (broken pairs) in progress - - Phys. Rev. 39C, 1066 (1989)

24. Evidence for important infuence of the Dynamical Pauli Effect on the behavior of nuclear $g$-factors with angular momentum, and on the behavior of the VMI modification of the nuclear moment of inertia as a function of particle number - To bc published

25. A new algebraic model of superdeformation which seems already to have the correct qualitative, and even quantitative, features in the analytical symmetry limit - To be published 
This is blatant propaganda, of course, but it is of the sort that we generally consider acceptable in physics because it is (for the most part) documented by literature references. Indeed, I hope that you will not take the preceding assertions at face value, but rather that you will go to the published papers and preprints and critically assess them for yourself.

The weight of the evidence cited above indicates that the FDSM provides a microscopic link between the common approaches to the structure of strongly collective nuclei and the spherical shell model: the FDSM is a truncation of the spherical sheil model problem which yields the Particle-Rotor Model and the Cranked Shell Model in the limit of large particle number, the Interacting Boson Model in the limit of large shell degeneracy (neglect of Pauli principle), and the Hartree-Fock Bogoliubov theory in the coherent state approximation. The situation is summarized in Fig.1.

Let's now turn to a more concrete examination of all of this. We begin by introducing the basic ideas of the FDSM in a form which will facilitate the later discussion. A more comprehensive description can be found in refs. 2 and 3.

\section{THE FERMION DYNAMICAL SYMMETRY MODEL}

The most useful way to view the FDSM is as a particular truncation of the spherical shell model. The FDSM truncation is unusual because it is a truncation according to principles of dynamical symmetry, a symmetry-dictated truncation, if you will. That is, in more normal truncations of the shell model space - and such trunctions are necessary at some level in any shell model calculation- the truncation is done according to energy considerations; the typical restriction of the shell model to a single major shell is one simple example of such an energy-dictated truncation. The truncation of the shell model space implied by the FDSM is qualitatively different. We adopt a point of view made familiar by the IBM approach to nuclear structure: collective modes correspond to dynamical symmetries in the underlying algebraic structure of many-body theories. However, in the FDSM we are going to look for these svmmetries directly in the fermion space, without recourse to boson degrees of freedom or mapping procedures; this is illustrated in Fig. 2.

The starting point for the FDSM is the Ginocchio $\mathrm{Model}^{4}$, where the angular momentum of a single-particle orbital is separated into an integer pseudo-orbital part $k$ and a hall-integer pseuospin part $i$. The coupling scheme for the Ginocchio model is shown in Table 1. In the Ginocchio coupling scheme the $k=1$ coupling may be termed $k$-active coupling, while the $i=3 / 2$ coupling may be termed $i$-active coupling. These couplings, and the normal coupling of the pairing model (which we will term $k=0$ coupling) are illustrated in Fig. 3.

These coupling schemes give rise to a nice group structure which can lead to dynamical symmetries. ${ }^{\dagger}$ In terms of monopole pair operators $S^{\dagger}$, quadrupole pair operators $D^{\dagger}$. and multiple operators $P^{r}$ of rank $r$, we may define the closed algebras shown in Table 2. The angular momentum labeling in these expressions corresponds to

$$
i_{1}+i_{2}=I \quad k_{1}+k_{2}=K \quad\left[b_{k_{1} i_{1}}^{\dagger} b_{k_{2} i_{2}}^{\dagger}\right]_{\mu_{K} \mu_{I}}^{K I}
$$

${ }^{1}$ A dynamical symmetry results if the Hamiltonian for a system can be written in terms of the invariants (Casimir operators) of a group and its subgroups. The dynamical symmetry chains listed here all end in the group $S O(3)$ corresponding to conservation of total angular momentum. 


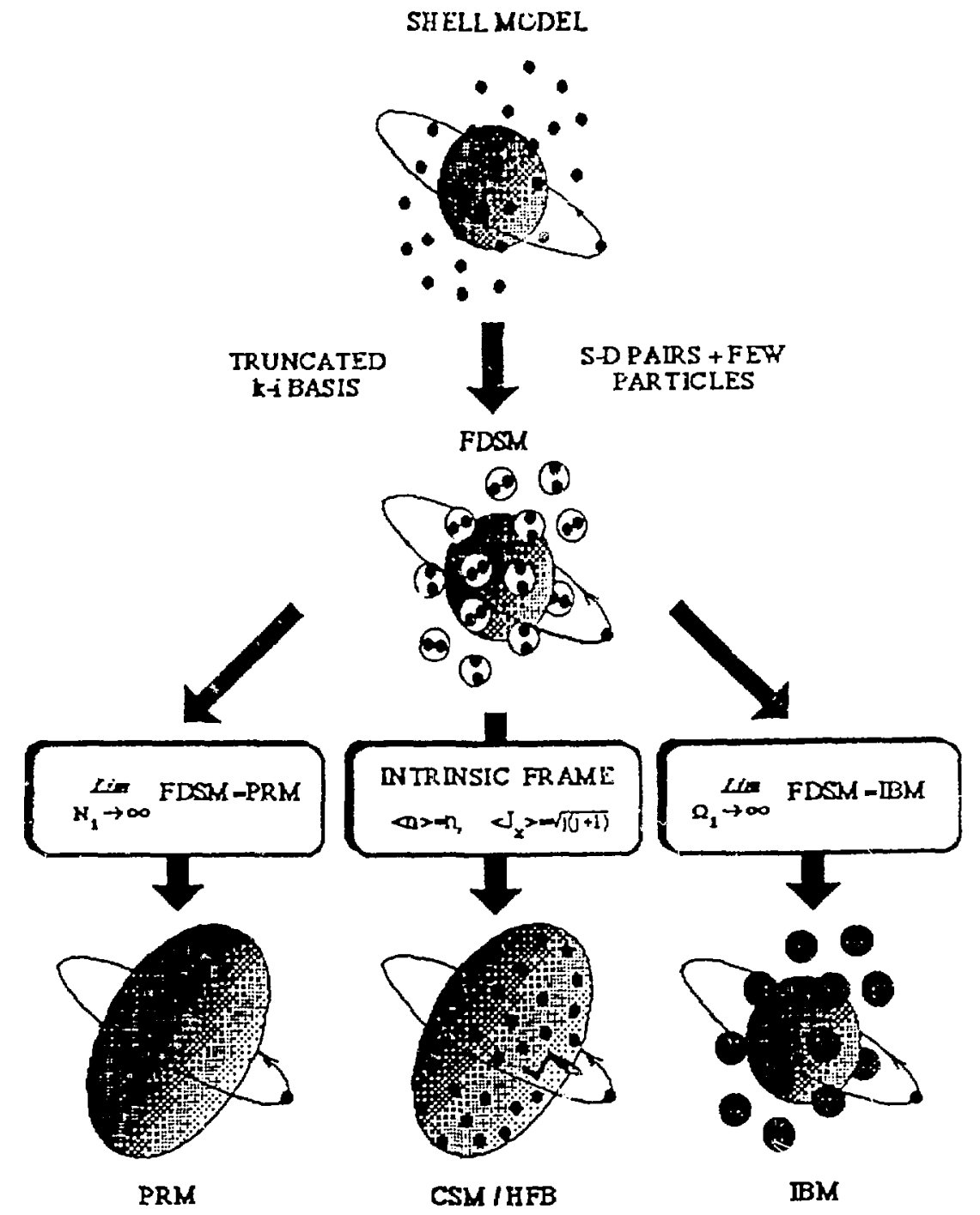

Figure 1. A schematic illustration of how the FDSM is related to standard models. Generally, the FDSM is derived from the spherical shell model through symmetry-dictated truncation. Then, geometry-based models represent the infinite particle number limit of the FDSM, algebraic boson models represent the infinite shell degeneracy limit of the FDSM, and HartreeFock modeis correspond to mean field approximations to the FDSM.

The $i$-active and $k$-active group structures give rise to dynamicail symmetries corresponding to the subgroup chains shown in Table 3 . The $k=0$ group structure corresponds to the well-known quasispin group, $\mathcal{S U}(2)$. By comparing matrix elements, these dynamical symmetry chains can be identified (in the limit of large particle number) with various geometrical collective modes, which are listed beside each dynamical symmetry:

The FDSM now results if we apply these coupling schemes to the spherical shell model in the following way. We require that for each major shell the normal-parity orbitals 


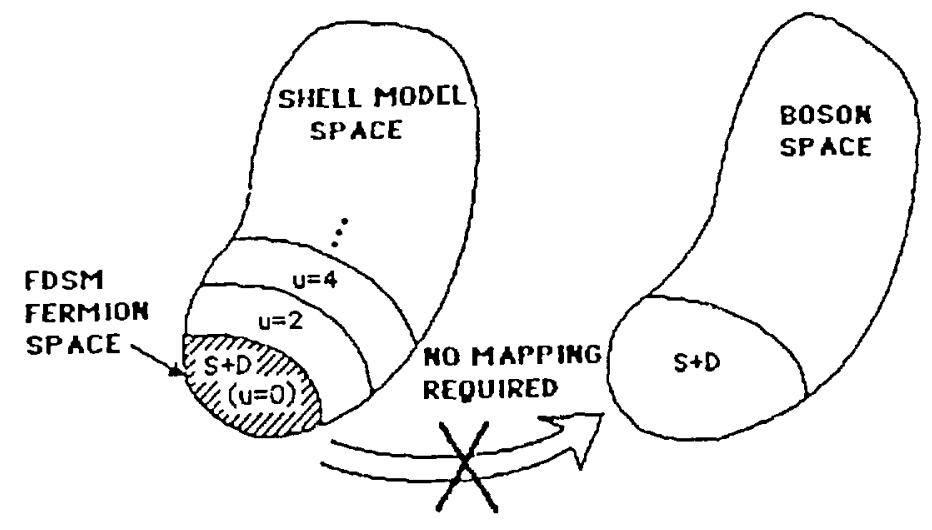

Figure 2. The FDSM is a symmetry-dictated truncation of the fermion shell model space. The inspiration for connecting dynamical symmetries witi collective modes is derived from algebraic boson models; however, the FDSM works at all times in the fermion space, so there is no need of a mapping procedure between the fermion and boson spaces. The FDSM truncates the shell model space with respect to the heritage quantum number $u$, which is the number of particles which do not form (collective) $S$ and $D$ fermion pairs. If all heritages are included, the FDSM model space becomes the original shell model space. For light nuclej this is computationally feasible, and the FDSM is the spherical shell model, at whatever level of truncation one wishes. For heavy nuclei in the middle of valence shells it is only possible to include low heritages in realistic calculations; however, there is considerable evidence that low-energy nuclear structure is dominated by such configurations. This is the algebraic formulation of the conventional idea that nuclear structure is well approximated by the geometrical collective modes plus a few broken pairs.

either be $k$-active $(k=1)$ or $i$-active $(i=3 / 2)$, and that this coupling reproduce exactly the normal-parity orbitals of the shell - no more, and no less. Such a reclassification appears to be unique, as illustrated in Fig. 4. The requirement that there be only a single abnormal-parity level in each shell requires that each abnormal-parity level correspond to $k=0$ coupling, as also illustrated in Fig. 4.

This unique assignment of coupling schemes to each shell, and the group structure already discussed for these coupling schemes, inplies that the FDSM associates particular dynamical symmetries, and therefore particular allowed collective modes, with each valence shell. The allowed symmetries are shown in the row labeled 'sym' in Fig. 4; the corresponding collective modes have been given above.

This shell-model reclassification leads to a unified, algebraic fermion description of collective motion, as illustrated in Fig. 5. I won't hother to describe the details of this somewhat formidable diagram, as they can be found in refs. 1,2. The essential point for our discussion is that in the FDSM the valence shell dictates the coupling scheme, which in turn defines the allowed dynamical symmetries. This leads to a rich variety of 


\section{GINOCCHIO MODEL}

Gencralized pseudo-orbit, pseudo-spin representation

$$
\begin{gathered}
\mathrm{j}=\mathrm{k}+\mathrm{i} \\
a_{j m}^{\dagger}=\sum_{m_{k} m_{1}}\left(k m_{k} i m_{i} \mid j m\right) b_{k m_{k} i m,}^{\dagger}
\end{gathered}
$$

MOTIVATION: Gencralize quasispin to include D-pairs

ASSUMPTION : $S$ and $D$ pairs play a crucial role in nuclear structure.

Ginocchio noted that two choices of $k$ and $i$ ensured that $S$ and $D$ (and only $S$ and $D$ ) pairs were formed:

$$
\begin{gathered}
i_{1}=i_{2} \equiv i=3 / 2 \\
k_{1}=k_{2} \equiv k=\text { Anything consistent with } \mathrm{j}=\mathrm{k}+\mathrm{i} \\
\mathrm{K}=\mathrm{k}_{1}+\mathrm{k}_{2}=0 \\
k_{1}=k_{2} \equiv k=1 \\
i_{1}=i_{2} \equiv i=\text { Anything consistent with } \mathrm{j}=\mathrm{k}+\mathrm{i} \\
\mathrm{I}=\mathrm{i}_{1}+\mathrm{i}_{2}=0
\end{gathered}
$$

Table 1. The Ginocchio Model. ${ }^{4}$

collective modes (corresponding to geometrical rotations and vibrations) with broken pairs of particles, or single particles, coupled to them.

\section{SOME PHYSICAL QUANTITIES CALCULATED WITH THE FDSM}

We now turn to some examples of applying the FDSM to the calculation of physical quantities. The FDSM program can be implemented at two general levels. In the symmetry limits one obtains analytical formulas and a systematic overview and classification of nuclear structure. However, real nuclei do not have perfect dynamical symmetries, so the second stage corresponds to using the symmetry limits as a starting point for numerical calculations with symmetry-breaking terms. Although both levels of investigation are being pursued, I will concentrate here on analytical calculations performed in the symmetry limits (or in perturbation theory around the symmetry limits). I do this 


\section{GTIOUP STRUCTURE}

(1) i-Active Coupling Scheme ( $i=3 / 2)$

GENERATORS: $S^{\dagger}, S, D_{\mu}^{\dagger}(i), D_{\mu}(i), P_{\mu}^{r}(i) \quad$ [Closed under $s o(8)$ Lie algebra]

$$
\begin{gathered}
S^{\dagger}=\sqrt{\Omega_{k 3 / 2} / 2}\left[b_{k 3 / 2}^{\dagger} b_{k 3 / 2}^{\dagger}\right]_{00}^{00} \\
D_{k}^{\dagger}(i)=\sqrt{\Omega_{k 3 / 2} / 2}\left[b_{k 3 / 2}^{+} b_{k 3 / 2}^{+}\right]_{0 \mu}^{02} \\
P_{\mu}^{r}(i)=\sqrt{\Omega_{k 3 / 2} / 2}\left[b_{k 3 / 2}^{\dagger} \tilde{b}_{k 3 / 2}\right]_{0 \mu \mu}^{0 r} \quad(r=0,1,2,3) \\
\Omega_{k i}=\sum_{j}(2 j+1) / 2=(2 k+1)(2 i+1) / 2 \quad \text { (pair degeneracy) }
\end{gathered}
$$

(2) k-Active Coupling Sclieme $(k=1)$

GENERATORS: $S^{\dagger}, S, D_{\mu}^{\dagger}(k), D_{\mu}(k), P_{\mu}^{r}(k)$ [Closed under $s p(6)$ Lie algebra ]

$$
\begin{aligned}
S^{\dagger} & =\sqrt{\Omega_{1 i} / 2}\left[b_{1 i}^{\dagger} b_{1 i}^{\dagger}\right]_{00}^{00} \\
D_{\mu}^{\dagger}(k) & =\sqrt{\Omega_{1 i} / 2}\left[b_{1 i}^{\dagger} b_{1 i}^{\dagger}\right]_{\mu 0}^{20} \\
P_{\mu}^{r}(k) & =\sqrt{\Omega_{1 i} / 2}\left[b_{1 i}^{\dagger} \tilde{b}_{1 i}\right]_{\mu 0}^{r 0} \quad(r=0,1,2)
\end{aligned}
$$

(3) Monopole Pair Coupling $(k=0)$.

GENERATORS: $\mathcal{S}^{\dagger}, \mathcal{S}, \mathcal{P}^{0} \quad$ [Closed under $s u(2)$ Lie algebra ]

$$
\begin{aligned}
& \mathcal{S}^{\dagger}=\sqrt{\Omega_{j_{0}} / 2}\left[b_{0 j_{0}}^{\dagger} b_{0 j_{0}}^{\dagger}\right]_{00}^{00} \\
& \mathcal{P}^{0}=\sqrt{\Omega_{j_{0}} / 2}\left[a_{j_{0}}^{\dagger} \tilde{a}_{j_{0}}\right]_{0}^{0}
\end{aligned}
$$

Table 2. Group structure of the Ginocchio and pairing models.

for two reasons. First, this illustrates the basic points that I wish to leave you with in the simplest fashion; second, we will find that already in the symmetry limit the results are quantitatively in agreement with data for a variety of physical observables.

Before discussing some specific calculations, there is one conceptual and practical point which may have been bothering you, and which requires some clarification. The discussion in the previous section has assumed a single bind of nucleon, and it pre- 


\section{DYNAMICAL SYMMETR.IES}

(1) i-Active Coupling $(i=3 / 2)$

$\begin{array}{ll}S O(8) \supset S O(5) \times S U(2) \supset S O(5) \supset S O(3) & \text { (Vibrator) } \\ S O(8) \supset S O(6) \supset S O(5) \supset S O(3) & (\gamma \text {-Unstable Rotor) } \\ S O(8) \supset S O(7) \supset S O(5) \supset S O(3) & \text { (Vibrator) }\end{array}$

(2) k-Active Coupling $(k=1)$

$$
\begin{array}{|lc|}
S p(6) \supset S U(3) \supset S O(3) & \text { (Axially-Symmetric Rotor) } \\
& \text {. } \\
S p(6) \supset S U(2) \times S O(3) \supset S O(3) & \text { (Vibrator) }
\end{array}
$$

(3) Monopole pair coupling $(k=0)$

$$
\text { SU(2) (Quasispin Symmetry) }
$$

Table 3. FDSM dynamical symmetries.

sumably has not escaped your attention that nuclei contain both valence protons and neutrons. Furthermore, there is widespread agreement that quadrupole collective modes are primarily a consequence of the quadrupole-quadrupole interaction between neutrons and protons. How then do $n-p$ interactions enter the FDSM?

The complete answer to this question would require more space than is available here; it has already been discussed to some degree in refs. 1 and 2, and will be discussed fully in a review article currently in preparation. ${ }^{5}$ Here, I will make only the following qualitative remarks. Generally, we assume a wavefunction which is a product of neutron and proton wavefunctions, and a Hamiltonian which contains an interaction term $H_{n p}$ which couples the neutrons and protons strongly through an $n-p$ quadrupole interaction. In the simplest calculations we assume that the product wavefunction is symmetric for the lowest-lying states. In some situations, such as in the actinides where the neutrons and protons have the same highest symmetry, it is possible to obtain a dynamical symmetry corresponding to a coupled neutron-proton system. For example, well-deformed actinides can be described by the syinmetry

$$
S U(3)^{\pi} \times S U(3)^{\nu} \supset S U(3)^{\pi+\nu},
$$

where $\pi$ and $\nu$ denote protons and neutrons respectively. In other cases, the neutrons 


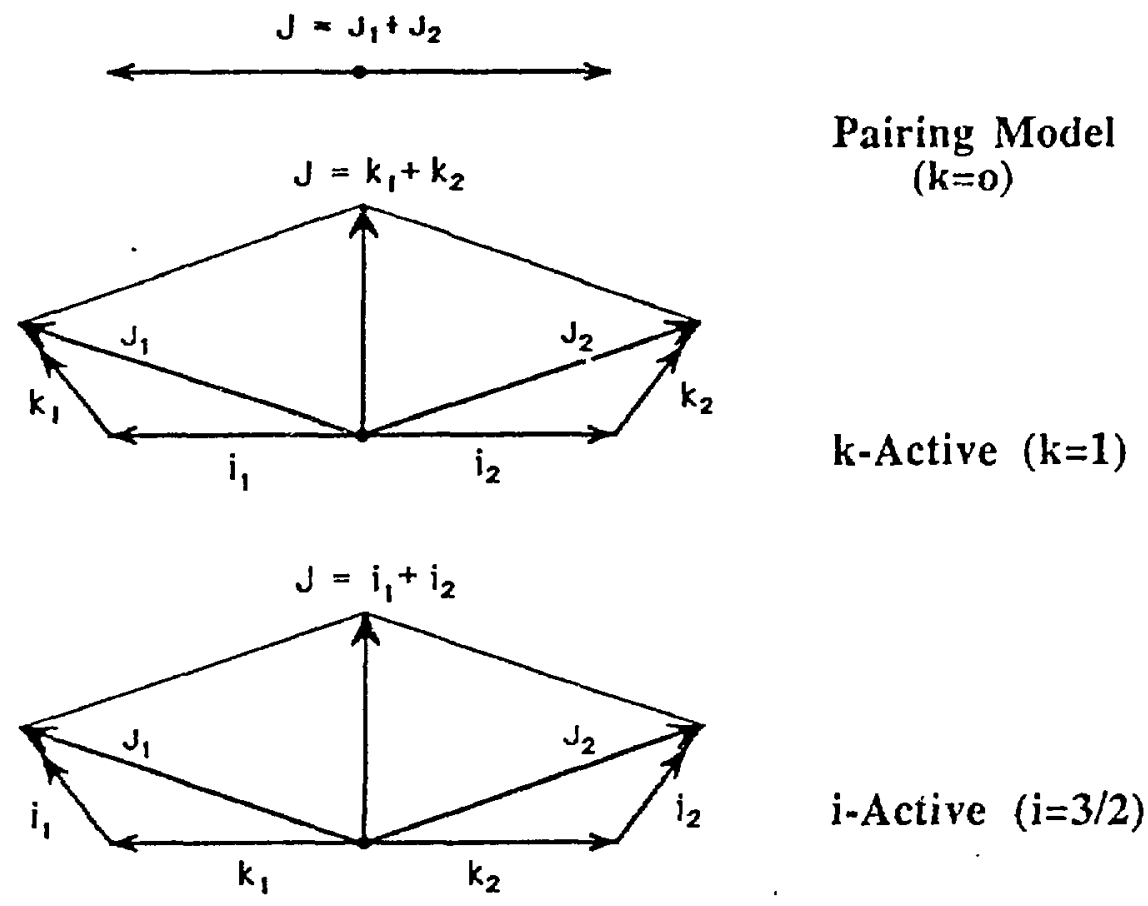

Figure 3. Illustration of the basic pair coupling schemes used in the Fermion Dynamical Symmetry Model for heritage-zero configurations. The $k$-active and $i$-active schemes are used for the normal-parity orbitals in the collective $S-D$ subspace. The $k=0$ coupling scheme is used for the heritage-zero sector of the abnormal parity orbital space. In each diagram the part of the angular momentum coupled to zero contribution to the pair angular momentum is termed the inert part of the single-particle angular momentum; the part coupled to finite angular momentum for the pair is termed the active part of the single-particle angular momentum. For configurations where the heritage is non-zero, the inert part of the angular momentum may be recoupled to a non-zero resultant angular momentum, which has the physical interpretation of the angular momentum of broken pairs. This angular momentum of broken pairs then couples to the active angular momentum of the pairs to give the total angular momentum in configurations with non-zero heritage.

and protons cannot be coupled like this, and there is no dynamical symmetry. This is the case for the rare-earths's, where neutrons are $S p(6)$ and protons are $S O(8)$. However, numerical calculations indicate that even when formally there is no dynamical symmetry for the neutron-proton system, there often is a practical dynamical symmetry. For example, numerical studies of well-deformed rare-earth nuclei indicated that they behave like the FDSM $S U(3)$ symmetry limii for many properties. ${ }^{6}$

Let's begin our survey of some FDSM calculations by looking at the FDSM calculation of ground-state masses in the actinide region. The details may be found in ref. 7 , and we present the results in Fig. 6. Even thought the FDSM calculation is analytical, it is seen to fit the 340 masses with 13 adjustable parameters and a r.m.s. error of 0.23 


\begin{tabular}{|c|c|c|c|c|c|c|c|c|}
\hline No. & 1 & 2 & 3 & 4 & 5 & 6 & 7 & 8 \\
\hline$n$ & 0 & 1 & 2 & 3 & 34 & 45 & 556 & $\begin{array}{lll}6 & 6 & 7\end{array}$ \\
\hline $\bar{k}$ & $\overline{0}$ & 1 & 1 & 0 & 10 & 2 & 110 & 10 \\
\hline$i$ & $\frac{1}{2}$ & $\frac{1}{2}$ & $\frac{3}{2}$ & $\frac{7}{2}$ & $\frac{3}{2} \quad \frac{9}{2}$ & $\begin{array}{ll}\frac{3}{2} & \frac{11}{2}\end{array}$ & $\frac{1}{2} \quad \frac{7}{2} \quad \frac{13}{2}$ & $\begin{array}{lll}\frac{3}{2} & \frac{9}{2} & \frac{15}{2}\end{array}$ \\
\hline 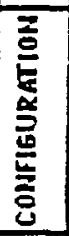 & $s_{1 / 2}$ & $\begin{array}{l}\mathrm{P}_{1 / 2} \\
p_{3 / 2}\end{array}$ & $\begin{array}{l}s_{1 / 2} \\
d_{3 / 2} \\
d_{5 / 2}\end{array}$ & $f_{7 / 2}$ & $\begin{array}{l}p_{1 / 2} \\
p_{9 / 2} \\
p_{3 / 2} \\
f_{5 / 2}\end{array}$ & $\begin{array}{l}s_{1 / 2} h_{11 / 2} \\
d_{3 / 2} \\
d_{5 / 2} \\
g_{7 / 2}\end{array}$ & $\begin{array}{c}p_{1 / 2} f_{5 / 2} i_{13 / 2} \\
p_{3 / 2} f_{7 / 2} \\
h_{g / 2}\end{array}$ & $\begin{array}{l}s_{1 / 2} g_{7 / 2} j_{15 / 2} \\
d_{3 / 2} g_{9 / 2} \\
d_{5 / 2} i_{11 / 2}\end{array}$ \\
\hline$\frac{\sum}{i s}$ & & & $G_{6} G_{8} G_{3}$ & & ${ }_{6} \mathbb{G}_{8}$ & $\mathbf{G}_{8}$ & $G_{6}$ & $G_{6}$ \\
\hline$\Omega_{0}$ & 0 & 0 & 0 & 0 & 5 & 6 & 7 & 8 \\
\hline$\Omega_{11}$ & 1 & 3 & $\overline{6}$ & 4 & 6 & 10 & 15 & 21 \\
\hline$n$ & 2 & 8 & 20 & 28 & 50 & 82 & 126 & 184 \\
\hline
\end{tabular}

$\mathrm{G} \sigma=S p 6 \times S U 2$

$\mathrm{G} 8=\mathrm{SO} 8 \times \mathbb{S U Z}$

$\mathrm{G} 3=\mathrm{SU} 3 \times \mathrm{SO} 6 \times \mathbb{S U 2}$
( k-active)

( $\mathrm{i}$-active )

(k/i-active)

Figure 4. FDSM reclassification of the shell model. The first two rows give the shell number and the oscillator quantum number. The row labeled 'configuration' gives the normal shell-model classification of orbitals. Rows 3 and 4 reclassify according to the FDSM quantum numbers $k$ and $i$. The quantities $\Omega_{0}$ and $\Omega_{1}$ are pair degeneracies of the normal and abnormalparity orbitals of the shell respectively. Normal-parity orbitals of a shell are either $k$-active $(k=1)$ or $i$-active $(i=3 / 2)$, or (in light nuclei) $k / i$-active ( $k=1$ and $i=3 / 2$ ); abnormal-parity orbitals are required to be $k=0$ (and therefore $j=i$ ) by the constraint that there be only one abnormal-parity orbital within a shell. The group structure implied for each valence shell is shown in the row labeled 'sym'.

$\mathrm{MeV}$.

In Fig. 7 we show an FDSM calculation of the $B\left(E 2,2^{+} \rightarrow 0^{+}\right)$for even-even nuclei in the rare-earth region. This result I consider to be rather remarkable, since it is again obtained analytically, and only a single overall scale parameter is adjusted - the shape of the curve is completely fixed by the theory! A similar quality description has been obtained for actinide nuclei, and for nuclei in the 50-82 shells: we find that all heavy even-even nuclei can be described with a quality comparable to that in Fig. 7 using only 3 adjustable parameters, which just set the scale of the $B(E 2)$ 's (the details may be found in ref. 9).

In Fig. 8 we show an example of another analytical FDSM calculation - in this case the behavior of $B(E 2)$ values at high angular momentum. Here we see the ability of the FDSM to reproduce the measured loss of $E 2$ collectivity at high angular momentum, 


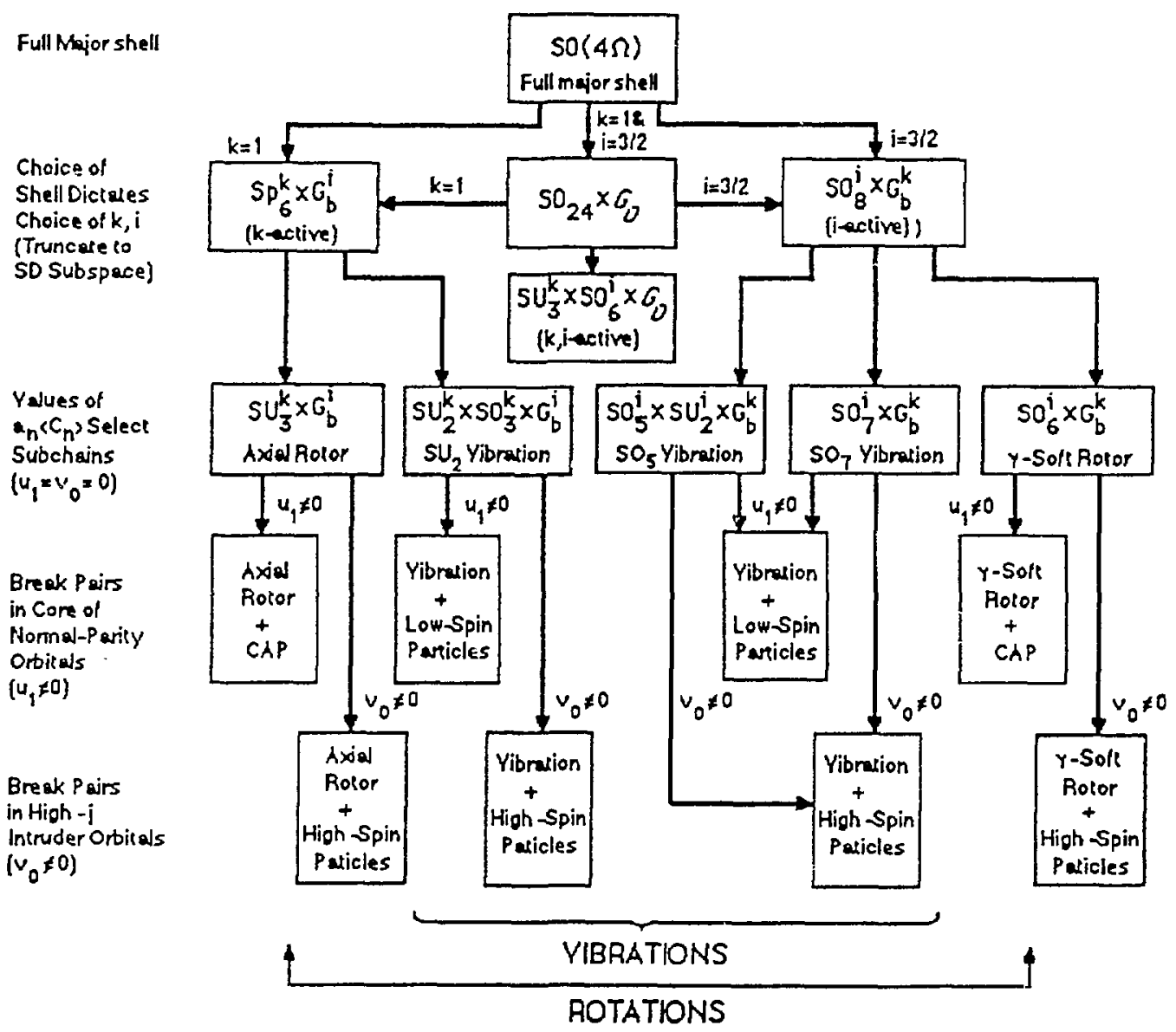

Figure 5. Overall group structure of the FDSM within a single major shell. Details may be found in refs. 1 and 2. CAP stands for Coriolis antipairing, and $G_{b}^{k, i}$ stands for the group structure associated with the abnormal-parity orbitals.

even when the moment of inertia stays relatively constant as in the case of ${ }^{166} \mathrm{Yb}$. This is because the essential loss of collective strength in the FDSM is a dynamical effect, not a shape change (the shape can change too, but that is a separate effect); thus, the $B(E 2)$ can change while the moment of inertia remains almost constant. The details of this calculation may be found in ref. 10 .

\section{THE DYNAMICAL PAULI EFFECT}

The results presented in Figs. 6-8 all have contained within them the action of a very important principle of collective motion which we term the Dynamical Pauli Effect.

DYNAMICAL PAULI EFFECT. A constraint on allowed representations of a dynamical symmetry brought about by the competition between building collectivity and the Pauli principle which is more restrictive than that imposed by the particle-hole symmetry.

An example is shown in Table 4: in the FDSM $S U(3)$ symmetry there are various representations near midshell which are forbidden by the Pauli principle; thus, $S U(3)$ 

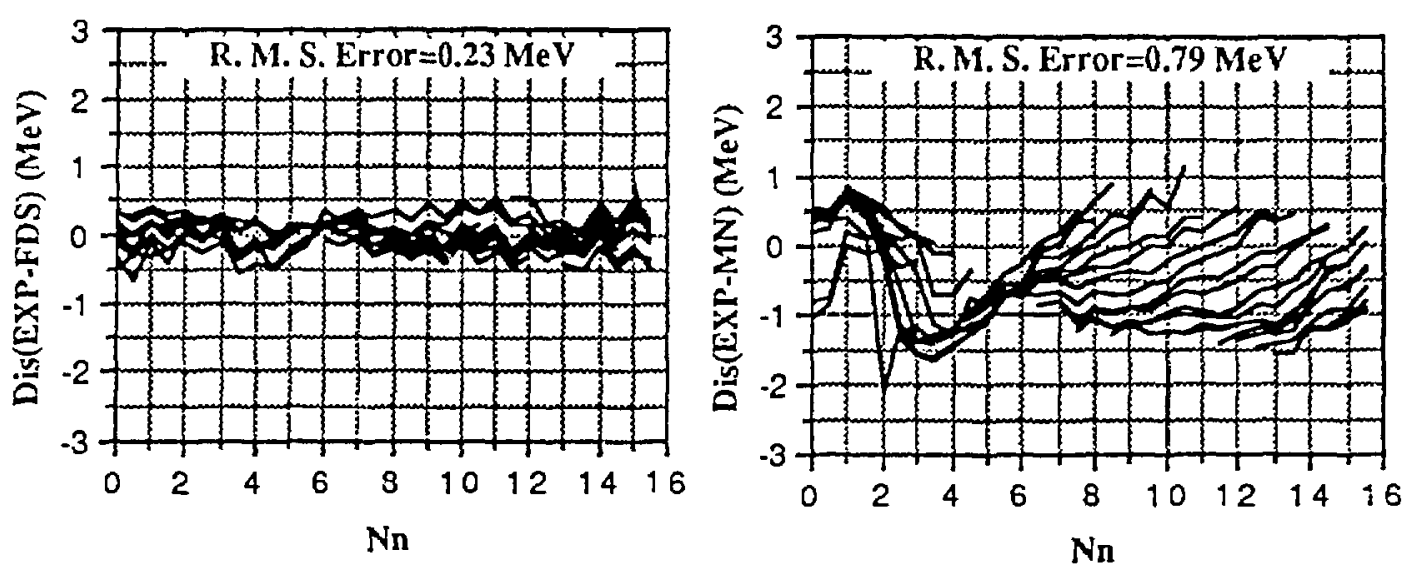

Figure 6. Calculated ground-state masses of actinide nuclei. The difference between theoretical and experimental masses is plotted as a function of valence neutron pair number $N_{n}$. The FDSM calculation on the left is analytical, and uses 13 adjustable parameters to fit the 340 known masses in this region with a root-mean-square deviation of $0.23 \mathrm{MeV}$. A corresponding calculation using the Strutinsky method (Møller and Nix, ref. 8) is shown on the right. The details of the FDSM calculation may be found in ref. 7 .

collectivity is restricted near midshell. This is a property of a group-theoretical solution of a fermion many-body system, but it has a simple physical interpretation. Building quadrupole collectivity requires maximal overlap of valence particles with an attractive effective interaction. As the shell is initially filled there are many free orbitals and this can be done without undue complaint from the Pauli principle. However, as the shell begins to be more filled the desire to build collectivity comes into conflict with the Pauli principle. This difficulty is exacerbated for collective states relative to singleparticle states because the required correlations for collective states can't be generated by putting particles into arbitrary orbitals.

Thus, collectivity will be suppressed by the Pauli principle at some percentage of shell filling. Where this occurs is a property of the details of the collective mode; that is, it is a property of the particular dynamical symmetry. For example, in the FDSM $S O(6)$ symmetry this happens at midshell (for the normal-parity orbitals). Since this is just the ordinary particle-hole symmetry, this causes no effects distinguishable in the low-lying states from that expected for the Pauli principle for independent particle motion. In this case we say there is no dynamical Pauli effect. However, for the $S U(3)$ symmetry the situation is different. There the collectivity begins to be suppressed when the normal-parity shell is $1 / 3$ full, not at midshell, and there is a dynamical Pauli effect between $N_{1}=\Omega_{1} / 3$ and $N_{1}=2 \Omega_{1} / 3$.

The suppression of collectivity near midshell displayed in Fig. 7 is a consequence of such a dynamical Pauli effect. The calculations indicate that the derivative curve shown in the lower part of Fig. 7 should pass through zero at $N_{n}=9, N_{n}=14$, and $N_{n}=17$, as a consequence of this dynamical Pauli effect (corresponding to the predicted peaks and valleys in the upper figure). The data fluctuate, but the average behavior is clearly in agreement with this prediction (which 1 remind you has no adjustable parameters available to shift the zeros). On the other hand, if one makes the same sort of plot, but with respect to the proton number, the collectivity peaks near normal-parity midshell, 

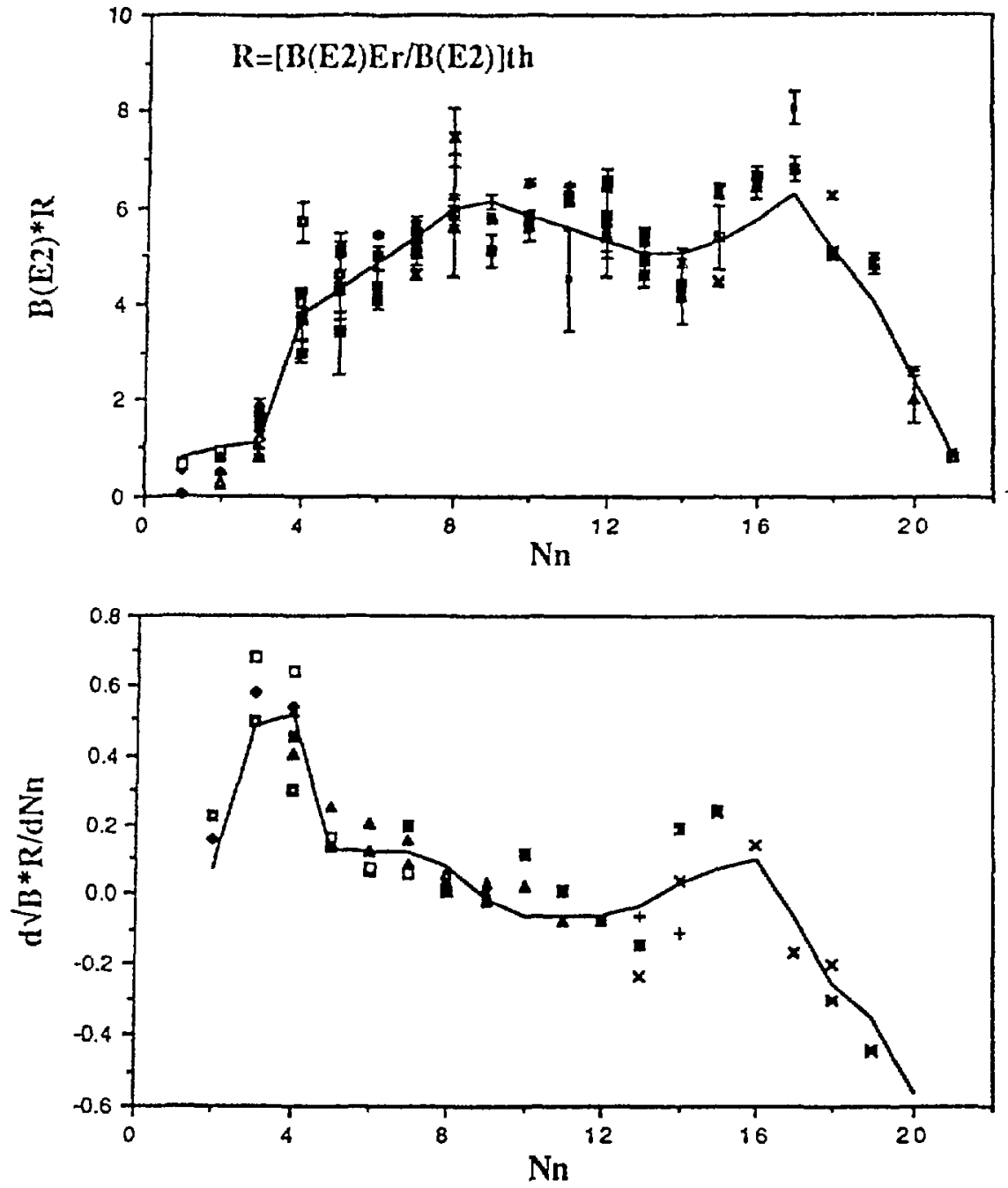

Figure 7. FDSM calculation of $B\left(E 2,0^{+} \rightarrow 2^{+}\right)$values for even-even nuclei in the rare-earth region as a function of neutron pair number, $N_{n}$. The original calculations are described in ref. 9. For ease in assimilating the results we have shown the data in the following way: In the top figure the data has had the theoretical proton number dependence scaled out using the FDSM. Thus, the deviation of the data from the line (FDSM calculation for $E_{r}$ isotopes) is a measure of the quality of the theory. There is only one adjustable parameters in the FDSM calculation; it corresponds essentially to an effective charge, so it can change only the scale: the shape of the FDSM curve is fixed by the theory, with no adjustable parameters. To emphasize the shape of the particle-number dependence, we show in the bottom figure the derivative of the upper figure with respect to the neutron pair number. Here too, the shape of the curve is parameter-free; only the overall scale is adjustable. The suppression of $B(E 2)$ values near midshell in the upper figure is an example of the Dynamical Paulj Effect. 

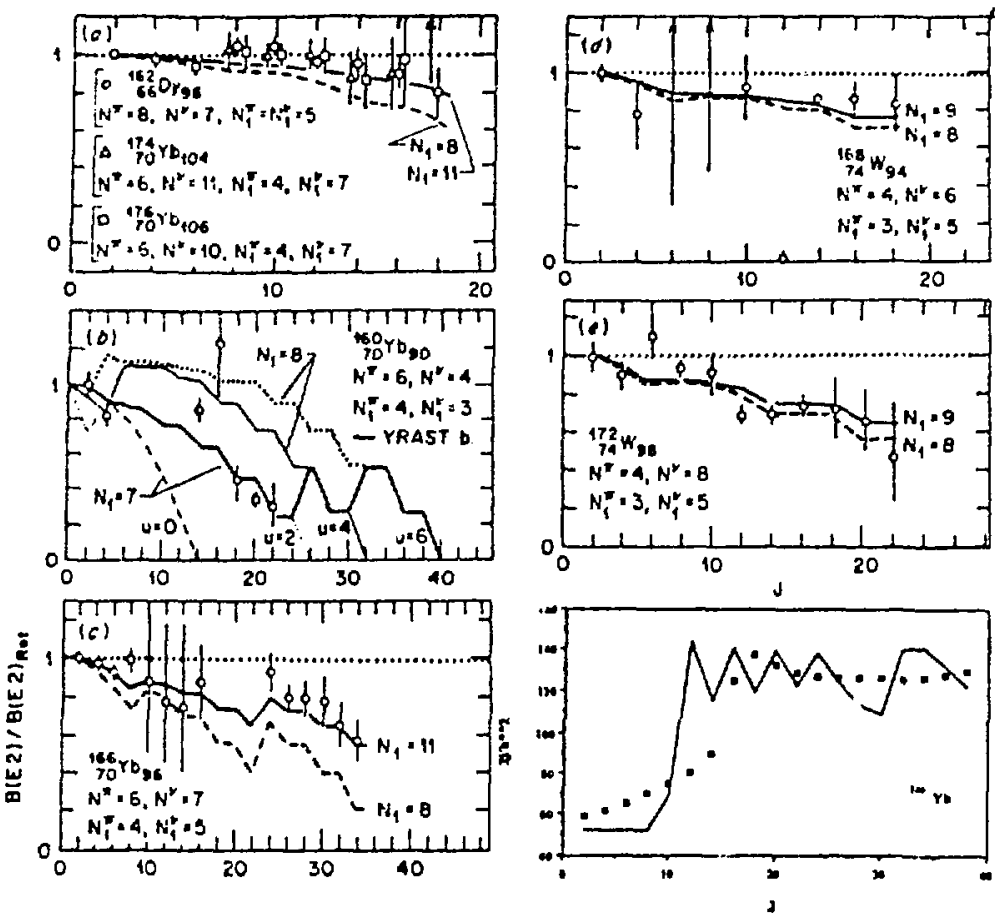

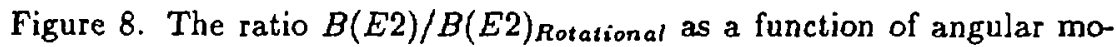
mentum for several rare-earth nuclei is shown in figures (a) - (e). The curves are two FDSM calculations with slightly different assumptions about distribution of particles in the valence shells. An $S U(3)$ symmetry was assumed, and the FDSM results are analytical; details may be found in ref. 10. Figure (b) exhibits a complete yrast band termination in the calculation near spin 40; in figure (b) only, the heavy line represents the yrast sequence and the lighter lines are non-yrast. The bottom right figure shows a corresponding FDSM calculation of the moment of inertia for ${ }^{166} \mathrm{Yb}$ as a function of angular momentum. This figure illustrates that in the microscopic FDSM theory the moment of inertia may remain nearly constant, even when there is a significant change in the $B(E 2)$ value as a function of angular momentum. The local fluctuations of the moment of inertia seen in this figure are a property of the dynamical symmetry limit. Realistically, small symmetrybreaking terms in the Hamiltonian would tend to smooth the fluctuations which appear above angular momentum 10.

as expected since there is no dynamical Pauli effect for the $S O(6)$ protons.

These dynamical Pauli effects are found in many properties of nuclei, two notable ones being $B(E 2)$ values and ground-state masses (effects in the spectrum will be discussed in \$6). In general, one finds that the inclusion of the explicit fermion nature of the generators of the dynamical symmetry - for example, the effect of the Pauli principle - improves the range of validity of the dynamical symmeties relative to those for pure 


\section{DYNAMICAL PAULI EFFECT}

The DYNAMICAL PAULI EFFECT is a systematic Pauli suppression of collectivity because of restrictions on allowed representations of a dynamical symmetry

EXAMPLE: FDSM $S p(6) \supset S U(3)$ Symmetry

The $S U(3)$ representation $(\lambda, \mu)=\left(2 N_{1}, 0\right)$ is Pauli forbidden when $N_{1}>\Omega_{1} / 3$.

$$
\left.\left(2 N_{1}, 0\right) \longrightarrow\left(2 N_{1}-4,2\right) \ldots\right]
$$

EXAMPLE: FDSM $S O(8) \supset S O(6)$ Symmetry

NC DYNAMJCAL PAULJ EFFECT; the usual lowest energy representation is allowed until $N_{1}=\Omega_{1} / 2$ (the normal particle-hole symmetry).

Table 4. The Dynamical Pauli Effect for FDSM $S p(6)$ symmetry.

boson models. ${ }^{t}$ For example, the pure $S U(3)$ boson theory fails to properly describe $B(E 2)$ values above angular momentum $\approx 10 \mathrm{k}$, but the $S U(3)$ limit of the FDSM, because it incorporates fermion degrees of freedom, can describe electromagnetic transitions to much higher angular momentum (Fig. 8). Therefore, an important qualitative FDSM result is that dynamical symmetries appear to be more prevalent in nuclei than would be thought from simple boson models, provided the generators of the symmetry incorporate the richer physics implied by the fermion nature of the problem.

\section{CRITICAL REVIEW OF OBJECTIONS TO THE FDSM}

I have presented a few examples of the power of the FDSM to describe physical observables in an economical manner in the previous section. These are only representative examples; there are many more that we could discuss if space allowed (see the summary in $§ 2)$. As in any theory which employs a new approach, there are criticisms, some a result of misunderstanding, but some of a more substantial nature. In this section I would like to briefly discuss some of these. In most cases I will only have space to sketch the objection, and my reply. A more complete discussion of all of this is in preparation. ${ }^{5}$

(1) Isn't the separation of the normal and abnormal-parity orbitals into a

'By pure boson models I mean the boson dynamical symmetries without Pauli factors generated by additional procedures such as boson mapping. If one does mapping, or boson expansions, it is of course possible to bring the Pauli effects into a boson theory at some level of approximation. 
direct product struct ure artificial? This separation presumably is artificial at some level. It is implemented in the FDSM as a mathematical convenience so that simple dynamical symmetries emerge. However, there are strong physical reasons to believe that the normal and abnormal-parity orbitals enter low-lying collective nuclear structure on very different footings. For example, consider the Nilsson model. The high- $j$ abnormal parity orbitals remain largely unmixed, even at normal nuclear deformations; this is easily seen by consulting the Nilsson wavefunctions, or by noting that the abnormal parity orbitals in a Nilsson diagram exhibit little curvature with deformation. ${ }^{\dagger}$ On the other hand, the normal-parity orbitals are strongly mixed at larger deformation, as seen from their wavefunctions, or their curvature in the Nilsson diagram.

This difference in behavior is associated with the removal of the abnormal-parity orbital from its fellows by the spin-orbit interaction: it has no nearby orbitals of the same parity with which to mix. As a consequence, the abnormal-parity orbital tends to behave more like a shell-model orbital at large deformation than the abnormal parity orbitals. This is precisely what is suggested by the FDSM quasispin symmetry for the abnormal-parity orbital (implying a dominance of pairing), and $S p(6)$ or $S O(8)$ symmetry for the normal-parity orbitals (implying the possibility of a large role for multipole interactions).

Finally, we note that a large amount of data is correlated by the FDSM with the assumption that in the $S U(3)$ limit the normal parity valence particle number $n_{1}$ and the abnormal-parity valence particle number $n_{0}$ are separately conserved, and that deformation systematics depend primarily on $n_{1}$. For example, the $B(E 2)$ systematics in Figs. 7 and 8 , and the mass systematics in Fig. 6, which reproduce the data at a level comparable with much more complicated traditional calculations, all employ this assumption.

Of course, the coupling between the normal and abnormal-parity orbitals does matter in realistic cases. For example, the odd-mass spectrum for the unpaired particle in an abnormal-parity orbital requires such a coupling, which breaks the dynan. cal symmetry and must be handled numerically. This we have described in if ref. 11 . However, our main requirement is not that dynamical symmetries describe nuclei perfectly, but that they be sufficiently good caricatures of nuclear structure that they are useful starting points for more sophisticated calculations. The FDSM separation of normal and abnormal-parity orbitals seems to fulfill this condition rather well.

(2) Don't the FDSM wavefunctions sometimes have poor overlap with "realistic wavefunctions"? Yes; and a good thing too! The FDSM must be understood as a microscopic theory of effective interactions in a truncated model space, and effective interactions always depend on the model space. The severely symmetry-truncated model space of the FDSM is certainly quite different from the model space of standard theories. Therefore, we may generally expect that the comparison of wavefunctions between the FDSM and other model spaces is meaningless. The relevant comparison is of matrix elements, for that is the best that we can measure in quantum mechanics. But it is quite clear that we can have $\left\langle\Psi_{1}|O| \Psi_{2}\right\rangle=\left\langle\Psi_{1}^{\prime}\left|O^{\prime}\right| \Psi_{2}^{\prime}\right\rangle$, even if $\Psi_{1} \neq \Psi_{1}^{\prime}$ and $\Psi_{2} \neq \Psi_{2}^{\prime}$ and $O \neq O^{\prime}$.

As we have seen in the preceding section, the FDSM gives a very good account of a variety of matrix elements (actually, squares of matrix elements in these examples).

Incidentally, it is important to distinguish the splitting of the abnormal parity orbitals, which is quite large, from the mixing of the abnormal parity orbitals with otler orbitals, which is quite small. 
Since the FDSM operators are chosen for their symmetry properties, they generally differ from corresponding operators in other theories. Thus, it is imperatwe that the FDSM wavefunctions differ from the wavefunctions in other theories if the matrix elements are to be equivalent, and the comparison of wavefunctions in different model spaces (see for example, refs, 12-13) is a red herring. The only relevant test of the FDSM is to compare with its matrir elements calculated using an effective interaction matched to the FDSM model space. The details of such a comparison constitute a long-term project, but the preliminary results are quite encouraging.

(3) Don't the FDSM symmetries work poorly in the sd shell? Actually, as we have empliasized in the caption to Fig. 2, the FDSM is identically the shell model in the $s d$ shell if all heritages are included. Since this is quite feasible technically for the light nuclei, this is more than an academic statement. Thus, the question is not whether the FDSM is "correct" in the sd shell (it is); the question rather is how large a heritage is required to approximate the correct physics in the sd shell. Just as in the discussion above, this comparison must not be sidetracked by comparisons of wavefunctions; it is the matrix elements which are relevant.

However, as can be seen in Fig. 4, and as wo have emphasized from the beginning, ${ }^{2,3}$ in the $s d$ shell the FDSM allows the nucleons to be both $k$ and $i$ actuve. In that case there is no a priori reason to prefer $k$ or $i$ active coupling, and the most reasonable assumption as a starting point is that both operate at the same time. Then the lowest symmetry is the full shell symmetry, which is $S O(24)$, and we would expect the collective motion in this shell to be complicated. It is only in the heavy nuclei where the symmetries simplify and the $k$-active or $i$-active coupling become unique.

(4) Doesn't the FDSM ignore $n-p$ interaction, which we all know to be the microscopic basis of deformation? This objection is based on a misconception. As we have already explained in $\$ 4, n-p$ interaction is essential to all the realistic FDSM calculations presented there. Indeed, the preliminary FDSM effective interaction indicates that in the truncated $S D$ subspace the effective $n-p$ quadrupole interaction is large and attractive, but the like-particle quadrupole-quadrupole interaction is weak, and may even be repulsive. The FDSM absolutely requires a strong $n-p$ quadrupale effective interaction to make sense of the properties of low-lying nuclear states.

$$
a b
$$

(5) Doesn't the: FDSM suggest that the normal-parity orbitals play no role in deformation systematics, and doesn't this contradict the conventional wisdom from the deformed shell model? This statement also is based on a misconception, and is the basis for some confusion. It is important to understand that the abnormal-parity orbital does play an enormous role in deformation systematics in the FDSM: agreement between FDSM calculations and data would be destroyed if the normal-parity orbital were removed. However, in the symmetry limits, the normal parity orbital plays a pivotal but indirect role; it doesn't influence the deformation so much by deforming itself as by serving as a kind of "sink" for particles driven from the normal-parity orbitals by the competing desires to maximize collectivity and at the same time satisfy the Pauli principle (the Dynamical Pauli Effect at work again). This allows the quadrupole collectivity to persist over a much broader range of nucleon number than would otherwise be possible, as required by the data, and has been likened to the "bufiering" role played by $f$ electron orbitals in the filling of the Lanthanide series 
of elements. ${ }^{\dagger}$ Of course, the abnormal parity orbital will also deform in the presence of the normal-parity deformed field (see ref. 11). Our results indicate that this is a secondary effect, however. The origin of normal deformation is primarily in the normalparity orbitals. (This is no longer true for superdeformation; hovever, that is another story which I have no time to tell).

Our detailed investigations cannot be reproduced here (see ref. 14, forthcoming), but there appears to be no basic contradiction between the FDSM and Nilsson pictures of deformation systematics. Indeed, we have shown how to derive the Nilsson model from the FDSM in the limit of large particle number, so it would be surprising if the result were otherwise.

(6) In the FDSM the rare-earth protons have $S O(8)$ symmetry, but the neutrons have $S p(6)$ symmetry; isn't that a bit strange? As we discussed in $\$ 4$, this implies that there is no dynamical symmetry in the rare-earth region when the $n-p$ interaction is turned on. However, numerical investigations indicate that pairing plus FDSM quadrupole interactions diagonalized in an $S O(8)^{\pi} \times S p(6)^{\nu}$ basis give $S U(2)$ like observables near closed shells, and $S U(3)$-like observables away from closed shells;" thus an effective dynamical symmetry is present in the rare-earth region. Furthermore, FDSM calculations of physical observables like spectra and $B(E 2)$ values agree well with the data for rare-earth nuclei. In particular, the $B(E 2)$ systematics displayed in Fig. 7 provide strong evidence for an approximate $S O(8)^{\pi} \times S p(6)^{\nu}$ structure for low-lying rare-earth states when analyzed as a function of proton number and neutron number (see ref. 9).

(7) You have emphasized the Dynamical Pauli Effect and its influence on properties like nuclear masses and electromagnetic transitions strengths; what about the effect on the spectrum? In the $S U(3)$ symmetry limit the disappearance of representations in midshell which was discussed in $\$ 5$ will also have a profound influence on the low-lying spectrum. Probably the most dramatic consequence is that the " $\gamma$-band" should drop to very low energy relative to the ground state. but not the " $\beta$-band".

To my knowledge, this does not happen in the rare-earth region. However, there are several things that one should keep in mind. First, these are pure symmetry-limit predictions which will be modified by symmetry-breaking in realistic cases. In the rareearth region this may be very important, because we have already argued that the different symmetries for the protons and neutrons in the rare earths mean there is no dynamical symmetry at all there. We have argued that there is a practical dynamical symmetry with respect to quantities like $B\left(E 2,2^{+} \rightarrow 0^{+}\right)$values; however, the numerical studies which show this have not yet been applied to a calculation of the spectrum in the Pauli forbidden region. In particular, we note that the assumption of completely symmetric states for the $n p$ system which we employed so succesfully for the ground and first (ground-band) $2^{+}$state in the rare earths is likely to break down for excited states. Therefore, the symmetry-limit prediction of the excited state spectrum in the rare-earths may fail, even if succesful predictions for the low-lying ground-state band survive. There appears to insufficient evidence to tell one way or the other so far.

The optimal place to see such effects would be the heavy actinides, since the FDSM predicts that deformed actinides will have $S U(3)$ symmetries for both protons and neutrons, and therefore a coupled $S U(3)$ symmetry will be favored by the $n-p$ quadrupole

TThis marvelous analogy was suggested to us by Bill Walters; I won't explain it further because this is a chemistry conference, and chemists are supposed to understand these things! 
interaction. Unfortunately, one begins to run out of stability at the $\Omega_{1} / 3$ boundary for both protons and neutrons in the actinides. However, for the little data available there is a substantial drop in the position of the $\gamma$ bandhead beyond $\Omega_{1} / 3$ for neutrons and/or protons (for example, in the heaviest $\mathrm{Fm}$ isotopes). This drop is about $50 \%$ relative to a straight-line extrapolation of data well before the $\Omega_{1} / 3$ boundary.

Therefore, I believe that the data is inconclusive in the rare-earths, until the effect of the expected symmetry-breaking is analyzed, and that there is a tantalizing hint of this effect in the actinides, but that we need data on several more nuclei to confirm it. I emphasize that these arguments don't prove that the FDSM is correct on this point; they just show that the jury isn't yet in. Speaking personally, I find it difficult to believe that the Dynamical Pauli Effect could slow up so clearly in quantities like transition rates and masses, and not show up in the spectrum for actinide nuclei; therefore, I consider the resolution of this matter to be one of the most important tasks facing the theory.

It appears that part of this resolution could come from better data in a region which requires special techniques and persistance on the part of the experimentalists. Thus we come to the topic of exotic spectroscopy.

\section{EXOTIC SPECTROSCOPY?}

I hope that I have at least made it plausible that the FDSM can, with only modest effort, describe a broad range of conventional nuclear properties. It then follows that the theory may be particularly well suited to the description of nuclei and properties far from the beaten path, for in those cases it may be necessary to go beyond the conventional assumptions. Clearly, the theory which is able to describe the conventional data with the simplest starting point is likely to fare best in this endeavor. Therefore, I will conclude this talk with a brief discussion of the FDSM and "exotic spectroscopy". I will define this loosely, with exotic ${ }^{\dagger}$ meaning 'out of the ordinary' and spectroscopy meaning 'the measurement of any properties of nuclear states'. Space limitations preclude more than a cursory listing of some of the ideas that have emerged from the FDSM; most of these are unpublished, and most have not yet been observed experimentally.

(1) The FDSM mass formulas predict the occurrence of stable superheavy elements which may differ somewhat in particle number from standard predictions, and which are bound by about $30 \%$ more than in standard predictions.

(2) The accuracy of the FDSM mass formula bodes well for predictions of masses far from stability. We are investigating possible astrophysical implications for this in $r$-process production of heavy elements.

(3) The FDSM predicts unusual effects in the collective motion associated with the Dynamical Pauli Effect for nuclei in the $Z \geq 100$ nuclei. These would appear in the excited vibrational band spectrum, and in quantities like electromagnetic transition rates, $g$-factors, and so on (see point $(\tau)$ in $\$ 6$ ).

(4) The FDSM suggests that there may be interesting things associated with the Coriolis Antipairing Effect (CAP) in a variety of nuclei: low-spin fluctuations in $B(E 2)$ values, disappearance of the CAP at certain nucleon numbers, anomalous quenching of twoparticle transfer strength at very low angular momentum, and previously unexpected angular momentum dependence of $g$-factors in the spin region below $10 \hbar$.

The dictionary offers "outlandish" as a definition; that has almost, but not quite, the desired connotation. 
(5) The FDSM suggests the possible existence of a new kind of nuclear state, the heritage isomer, which is an unusual many-body configuration for which "breaking" pairs lowers the energy.

(6) The FDSM suggests that an exotic kind of collective motion might develop in nuclei which have a number of like particles outside a closed shell, but very weak $n-p$ interaction. If the like-particle quadrupole-quadrupole effective interaction is weakly repulsive, as it may be, the $S U(3)$ representations might be inverted relative to their more common order. The resulting collective motion would be quite different from normal rotation for the low-lying states.

(7) The FDSM has been used to construct a model of superdeformation which seems to give a good account of the data, and which may allow a unified algebraic theory of superdeformations, fission isomers, and giant resonances.

\section{SUMMARY}

In summary, I hope that this introduction to the Fermion Dynamical Symmetry Model has driven home a basic point: dynamical symmetries which emphasize fermion degrees of freedom appear to be quite common in complicated many-body systems. This allows a fresh conceptual perspective on the nuclear structure problem; at the same time, the presence of the approximate dynamical symmetries suggests new ways to make practical microscopic calculations in these systems. Although the emphasis here has been on "normal" spectroscopy, it is clear that such an approach may be even more useful for "exotic spectroscopy". This is a fitting place to end, for it was our original motivation for gathering in this Symposium.

\section{REFERENCES}

1. C. L. Wu, D. H. Feng, X. G. Chen, J. Q. Chen, and M. W. Guidry, Phys. Lett. 168B, 313 (1986).

2. C. L. Wu, D. H. Feng, X. G. Chen, J. Q. Chen, and M. W. Guidry, Phys. Rev. 36C, 1157 (1987).

3. J. Q. Chen, X. G. Chen, D. H. Feng, C. L. Wu, J. N. Ginocchio, and M. W. Guidry, Phys. Rev. 40C, 2844 (1989).

4. J. Ginocchic, Ann. Phys. 126, 234 (1980).

5. C. L. Wu, J. Q. Chen, D. H. Feng, and M. W. Guidry, in preparation for Advances in Nuclear Physics.

6. H. Wu and M. Vallieres, Phys. Rev. 39C, (1989).

7. C. L. Wu, X. L. Han, Z. P. Li, M. W. Guidry, and D. H. Feng, Phys. Lett. 194B, 447 (1987).

8. P. Møller and J. R. Nix, Atomic Data and Nuclear Data Tables 39, 213 (1988).

9. D. H. Feng, C. L. Wu, M. W. Guidry, and Z. P. Li, Phys. Lett. 205B, 156 (1988).

10. M. W. Guidry, C. L. Wu, Z. P. Li, D. H. Feng, and J. N. Ginocchio, Phys. Lett. 187B, 210 (1987); and to be published.

11. H. Wu, D. H. Feng, C. L. Wu, Z. P. Li, and M. W. Guidry, Phys. Lett. 193B, 163 (1987).

12. P. Halse, Phys. Lett. 189B, 119 (1987).

13. P. Halse, Phys. Rev. 39C, 1104 (1989).

14. Z. P. Li, M. W. Guidry, C. L. Wu, and D. H. Feng, "The Origin of Deformation in the Nilsson Model and the Fermion Dynamical Symmetry Model". To be Published. 


\section{DISCLAIMER}

This report was prepared as an account of work sponsored by an agency of the United States Government. Neither the United States Government nor any agency thereof, nor any of their employees, makes any warranty, express or implied, or assumes any legal liability or responsibility for the accuracy, completeness, or usefulness of any information, apparatus, product, or process disclosed, or represents that its use would not infringe privately owned rights. Reference herein to any specific commercial product, process, or service by trade name, trademark, manufacturer, or otherwise does not necessarily constitute or imply its endorsement, recommendation, or favoring by the United States Government or any agency thereof. The views and opinions of authors expressed herein do not necessarily state or reflect those of the United States Government or any agency thereof. 